\title{
EL PAPEL DE LA TRANSFERENCIA INTERNACIONAL DE TECNOLOGÍA EN LOS SISTEMAS NACIONALES DE INNOVACIÓN DE LOS PAÍSES EN DESARROLLO
}

\section{THE ROLE OF INTERNATIONAL TECHNOLOGY TRANSFER IN THE NATIONAL INNOVATION SYSTEMS OF DEVELOPING COUNTRIES}

\author{
Larry Yumibanda Montiel, Mgs. \\ Magíster en Negocios Internacionales y Gestión de Comercio Exterior (Ecuador). \\ Profesor de la Facultad de Ciencias Económicas de la Universidad de Guayaquil y \\ Presidente del Colegio de Economistas del Guayas, Ecuador. \\ llarry.yumibandam@ug.edu.ec \\ Bladimir Jaramillo Escobar, Mgs. \\ Master en Docencia y Gerencia en Educación Superior (Ecuador). \\ Profesor e Investigador de la Facultad de Comunicación Social de la Universidad de \\ Guayaquil, Ecuador. \\ bladimir.jaramilloe@ug.edu.ec \\ Élita Luisa Rincón Castillo, Ph.D. \\ Doctora en Ciencias Económicas (Venezuela). \\ Profesora de la Escuela de Economía, Directora e Investigadora del Centro \\ Socioeconómico del Petróleo y Energías Alternativas, Facultad de Ciencias Económicas y \\ Sociales de la Universidad del Zulia, Venezuela. \\ rincon_elita@fces.luz.edu.ve
}

Carlos Alberto Añez González, Mgs. Magíster en Economía mención Macroeconomía y Política Económica (Venezuela). Profesor del Núcleo Costa Oriental del Lago, Investigador del Centro Experimental de Estudios Latinoamericanos "Dr. Gastón Parra Luzardo" de la Universidad del Zulia, Venezuela. caranez@luz.edu.ve

ISSN: 1390-9320, Vol. 7, No. 1, febrero 2020 


\section{ARTÍ́CULO DE REFLEXIÓN}

Recibido: 28 de noviembre de 2019.

Aceptado: 8 de enero de 2020.

\section{RESUMEN}

Una de las principales formas de difusión del conocimiento, desde el punto de vista del desarrollo productivo y de la capacidad de innovación, es la transferencia de tecnología. Esta investigación tiene como propósito analizar varios aspectos que afectan la oferta y la demanda de tecnología y determinan que su transferencia ocurra en un escenario cambiante. Se realizó un estudio reflexivo y de interpretación de autores relevantes en las variables objeto de estudio a partir de una metodología de investigación documental. Dentro de los resultados obtenidos se observa que los países menos desarrollados, los cambios tecnológicos que se producen son generados, mayormente, mediante la transferencia de tecnologías provenientes de los países más desarrollados, mientras la tecnología obtenida localmente tiene una menor participación. En este contexto, en particular en los países en vías de desarrollo, entre ellos los de América Latina, el término se refiere principalmente a la transferencia internacional de tecnología. Se concluye que, frente a una situación política marcada por recaídas en los procesos de integración económica y monetaria, se torna difícil llegar a materializar formas autónomas de generación, comercialización y compartición del "saber hacer" local-sectorial, las que constituyen la médula del progreso y expansión económica, pasos necesarios para el desarrollo económico sostenible.

Palabras clave: mercado de la tecnología, política tecnológica, transferencia internacional de tecnología, sistema nacional de innovación, países en desarrollo.

\section{ABSTRACT}

One of the main ways of disseminating knowledge, from the point of view of productive development and innovation capacity, is the transfer of technology. This research aims to analyze several aspects that affect the supply and demand of technology and determine 
that its transfer occurs in a changing scenario. A reflexive and interpretive study of relevant authors was carried out in the variables under study based on a documentary research methodology. Among the results obtained, it is observed that the less developed countries, the technological changes that are produced are generated, mainly, by the transfer of technologies from the more developed countries, while the technology obtained locally has a lower participation. In this context, particularly in developing countries, including those in Latin America, the term refers mainly to the international transfer of technology. It is concluded that, in the face of a political situation marked by relapses in the processes of economic and monetary integration, it becomes difficult to materialize autonomous forms of generation, commercialization and sharing of local-sector "know-how", which constitute the core of the economic progress and expansion, necessary steps for sustainable economic development.

Keywords: technology market, technology policy, international technology transfer, national innovation system, developing countries.

\section{INTRODUCCIÓN}

Durante las últimas décadas del siglo XX y lo transcurrido del XXI, la problemática asociada a la tecnología ha adquirido una gran importancia para las organizaciones que conforman la economía a nivel local, regional, nacional e internacional. Básicamente, este hecho surge debido a la aceleración del progreso y cambio tecnológico, fenómeno que lleva a un mayor número de innovaciones tecnológicas en un plazo de tiempo cada vez más reducido. Inevitablemente, el estudio de la tecnología debe llegar necesariamente a las universidades latinoamericanas, en concreto, a las facultades de ciencias económicas y sociales, a las facultades de ingeniería y a las facultades de ciencia y tecnología (Rincón, 2008, 2010).

Un tema de gran interés en este ámbito es la transferencia internacional de tecnología, la cual desempeña un papel clave en los sistemas nacionales de innovación que tienen un elevado componente de tecnologías importadas (Correa, 1994). Un aspecto central en las estrategias tecnológicas de los países en desarrollo es la adquisición eficiente de 
tecnologías y su adaptación a las condiciones específicas de la nación receptora. La naturaleza y el alcance de las diversas maneras de transferencia de tecnología se han modificado a través del tiempo como resultado de diversos factores que afectan el mercado internacional de la tecnología: su oferta y demanda.

En un mundo cada vez más interdependiente, los procesos mediante los cuales se realiza la transferencia de tecnología entre las naciones son de suma importancia. Para países en desarrollo, como los de América Latina, cuyos recursos tecnológicos son mucho más escasos frente a los del mundo desarrollado, esto es más evidente. En la historia socioeconómica de los países latinoamericanos, ha ido cambiando la óptica desde la cual se ha analizado la transferencia internacional de tecnología (Ávalos, 1994).

De manera muy general, es posible observar una transformación que va desde la denuncia a los daños económicos y sociales que acarrean la importación y uso de las tecnologías foráneas, hasta la visión que trata de integrar la adquisición de estas tecnologías a la estrategia de generación de capacidades propias por la vía de las estrategias de aprendizaje tecnológico. Lo anterior ha influido en las políticas y acciones que se han diseñado y puesto en práctica por los gobiernos, con respecto a la transferencia internacional de tecnología. Asimismo, se observa un énfasis en la utilización de la gestión tecnológica a nivel de la empresa para abordar este proceso.

Por otra parte, la transferencia internacional de la tecnología emerge como un tema de investigación importante en América Latina a finales de los años sesenta y cobra gran fuerza durante la década del setenta del siglo XX. En los años ochenta y noventa de ese siglo toma un nuevo giro, pero se mantiene su relevancia de primer orden. En la medida en que la producción de tecnología está fuertemente concentrada en pocos países y dado que la tecnología es un elemento clave cada vez mayor para el crecimiento económico, la competitividad y el comercio internacional, este tema seguirá teniendo pertinencia, sobre todo para los países en desarrollo, puesto que éstos, generalmente, no la generan por cuenta propia, sino que la adquieren de terceros. Por tanto, para el caso de estos países, 
hablar de las políticas de transferencia de tecnología, es hablar en gran medida de política tecnológica.

En la presente investigación se analizan varios aspectos que afectan la oferta y la demanda de la tecnología y determinan que su transferencia ocurra en un escenario cambiante. En primer lugar, se hace una revisión teórica de las categorías conceptuales transferencia internacional de tecnología y sistema nacional de innovación. En segundo lugar, se señala la metodología utilizada en la investigación. En tercer lugar, se establece el análisis de resultados a partir del papel que juega la transferencia internacional de tecnología en los sistemas nacionales de innovación de los países en desarrollo.

\section{REVISIÓN TEÓRICA}

En este apartado se hace una revisión teórica acerca de las categorías conceptuales que se analizan en esta investigación: la transferencia internacional de tecnología y el sistema nacional de innovación.

\subsection{Transferencia de tecnología: una perspectiva internacional}

Una de las principales formas de diseminación del conocimiento, desde el punto de vista del desarrollo productivo y de la capacidad de innovación, es la transferencia de tecnología, traducido en inglés como Technology Transfer (Lutteral, 2016). La idea de la transferencia de la tecnología surge a partir de que ningún país, ni ninguna empresa u organización, puede ser totalmente autosuficiente en lo que a tecnología se refiere (Escorsa y Valls, 2001).

\subsubsection{Definiciones de transferencia de tecnología}

En la literatura especializada, la noción de transferencia tecnológica se entiende de diversos modos (Ávalos, 1994). Por una parte, se emplea para identificar los procesos por medio de los cuales los conocimientos generados en las unidades de investigación aplicada

ISSN: 1390-9320, Vol. 7, No. 1, febrero 2020 
(laboratorios, centros e institutos tecnológicos) pueden llegar a ser utilizados por las empresas u organizaciones, a esto se le denomina transferencia de tecnología "vertical". Por otra parte, este término también puede ser utilizado para señalar los procesos mediante los cuales una tecnología diseñada para un determinado sector industrial es usada en otro distinto, a esta clase se le denomina transferencia de tecnología "horizontal".

Por consiguiente, la transferencia de tecnología se refiere a un conjunto de procesos que permiten que el flujo de conocimientos científicos, técnicos y empíricos entre las diferentes partes interesadas, como los gobiernos, el sector privado, las instituciones financieras, las instituciones de investigación o educación sean aplicados sistemáticamente a la elaboración de un producto o servicio (Pineda, Duarte, Ponce, Guzmán y Huaca, 2016).

En los países menos desarrollados, los cambios tecnológicos que se dan son generados en mayor medida mediante la transferencia de tecnología, proveniente de los países más avanzados, y la tecnología desarrollada localmente tiene una menor participación (Sánchez, 2016). En este contexto, en particular en los países en desarrollo, entre ellos los de América Latina, el término alude principalmente a la transferencia internacional de tecnología, el cual está referido al flujo de conocimientos que tiene lugar entre países, particularmente, el que ocurre entre los países en desarrollo y las naciones desarrolladas. La transferencia de la tecnología es un problema mucho más complejo que la simple difusión de innovaciones tecnológicas, si bien tal difusión es un medio clave de transferencia de tecnología (Martínez, 1994). Por lo tanto, en los países en desarrollo, la transferencia tecnológica, no ocurre tan frecuentemente, en comparación con el común proceso de comercialización de tecnología, que si se observa en los países desarrollados. Desde la perspectiva de los países en desarrollo, existe la Conferencia de las Naciones Unidas sobre Comercio y Desarrollo (UNCTAD, en inglés), quien en su publicación "Código internacional sobre la transferencia tecnológica" define la transferencia tecnológica como la transferencia de conocimiento sistemático para la elaboración de un producto, la aplicación de un proceso o la prestación de un servicio (Pineda et al., 2016). Asimismo, es importante señalar que la transferencia de la tecnología se maneja con base al régimen de protección de derechos de propiedad intelectual de la Organización Mundial del Comercio (OMC). De 
acuerdo con esta institución, los derechos de propiedad intelectual conceden al inventor (de la tecnología) el derecho a usarla de manera exclusiva durante un cierto período de tiempo; otorgándole con este fin la protección de los resultados de la inversión en el desarrollo de nuevas tecnologías y facilitando su transferencia (Pineda et al., 2016).

\subsubsection{Paquete tecnológico}

La transferencia de tecnología puede ser definida, como el traspaso de un paquete tecnológico o parte de él, desde una unidad u organización a otra, con el objeto de que esta última genere bienes y servicios (González, Clemenza y Ferrer, 2008). En este sentido, dada la conceptualización de la tecnología como un conjunto de conocimientos susceptible de uso productivo, se debe pensar la transferencia de tecnología en términos de un "paquete" integral que el proveedor le suministra al comprador.

En general, el paquete tecnológico "ideal” está compuesto por las siguientes piezas, según Ávalos (1994): i) uno o más módulos, integrado por tecnologías medular o periférica, los cuales pueden ser transmitidos por medio de documentación escrita, explicaciones principales, entrenamiento, asistencia técnica, entre otros; ii) autorización para utilizar varios derechos, conocimientos o activos; iii) bienes físicos, los cuales pueden tomar la forma de bienes de capital, intermedios o finales; iv) bienes intangibles (o blandos), los cuales pueden tener la forma de información escrita, programas de computación, transmisión oral, grabaciones, entre otros.

\subsubsection{Proceso de transferencia de tecnología}

Conceptualmente, la consolidación de la transferencia de tecnología involucra diferentes fases: absorción y aprendizaje, adaptación al medio ambiente y necesidades locales, la asimilación de las mejoras o adelantos posteriores, y finalmente, la generalización (Lutteral, 2016). 
a) La fase de absorción se corresponde básicamente con las actividades iniciales dedicadas al aprendizaje y aplicación de los conocimientos tecnológicos importados, más el mantenimiento de los equipos y su rendimiento a largo plazo.

b) La fase de adaptación a las condiciones locales puede comprender la tarea de desarrollo e innovación, permitiendo que la tecnología pueda ser ajustada a las necesidades locales, tanto del lado de la oferta, como de la demanda.

c) La fase de asimilación de adelantos posteriores significa que las mejoras aportadas por el nuevo conocimiento tecnológico incorporado no culminan cuando se efectúa la transferencia. A veces implican sólo la extrapolación de fenómenos o conocimientos existentes, pero en otros casos, implican la apertura de nuevas áreas de conocimiento, supuesto en el cual los operadores que pretenden dominarlo deberán enfrentarse con este nuevo fenómeno.

g) La fase de generalización se refiere a la posibilidad de incorporación de la tecnología por parte de todos los sectores que potencialmente se beneficiarían con dicha incorporación. Este proceso se concreta a través del contrato de transferencia tecnológica.

\subsubsection{Canales de transferencia de tecnología}

Los canales de transferencia de la tecnología son los medios entre los participantes particulares en el proceso (Gurbiel, 2002). Ellos incluyen las formas de adquirir la tecnología (compra, préstamo) y otros importantes factores relacionados con el proceso (flujos de personas, documentación, productos, capital). La transferencia tecnológica es conducida a través de diferentes canales y diferentes entes.

Dependiendo de las formas de crear y obtener la tecnología su transferencia puede ser considerada de tipo interno o externo (Gurbiel, 2002). La transferencia interna de tecnología es dirigida principalmente dentro de una misma unidad o entre sus afiliadas. Esta entidad actúa tanto como creador / innovador como usuario. El alcance del proceso interno está limitado por los recursos propios de investigación y desarrollo, así como de sus capacidades de aplicación. La transferencia tecnológica a lo externo descansa sobre recursos normalmente no relacionados con el comprador. 
Para Hoekman, Maskus \& Saggi (2005), la transferencia internacional de tecnología se lleva a cabo a través de los siguientes canales:

a) Comercio de bienes: puesto que el intercambio contribuye a la transferencia internacional de tecnología al facilitar la ingeniería local en reverso y el acceso a nueva maquinaria y equipo.

b) Inversión extranjera directa y concesionarios: aun cuando los efectos de ésta sobre los países menos desarrollados pueden ser ambiguos, varios estudios indican que predominan los efectos positivos. Las subsidiarias integradas ejercen un impacto positivo sobre el país receptor, a menudo acompañado por relaciones verticales «hacia atrás» y externalidades. c) Licencia: es una importante fuente de trasnsferencia internacional de tecnología para el mundo en desarrollo. Los contratos, normalmente, involucran la adquisición de derechos de producción o distribución y de la información técnica subyacente (know how).

d) Rotación de trabajadores y movimientos de personas: funcionan como medios de difusión tecnológica en casos donde las firmas locales no están muy a la zaga de las empresas transnacionales; paralelamente, un desafío de política para los países en desarrollo consiste en facilitar traslados temporarios de personas al exterior a la vez que alientan su retorno para asumir el desarrollo de negocios y la investigación tecnológica a nivel local.

\subsection{Sistema nacional de innovación}

El concepto de sistema nacional de innovación fue formulado originalmente para explicar el dinamismo tecnológico de los países desarrollados, en particular el caso de Japón, pero se ha extendido para ser aplicado a países en desarrollo o de reciente industrialización (Freeman, 1993; Rivera y Caballero, 2003). La innovación tecnológica es, por tanto, propia de cada país y se encuentra arraigada en su sistema nacional de innovación (Rincón, 2004). 


\subsubsection{Origen del sistema nacional de innovación}

El economista alemán Friedrich List (1789-1846) fue uno de los primeros impulsores de los sistemas nacionales de innovación, a partir de su libro Sistema nacional de economía política, publicado en 1841 (List, 1997). El propósito "era proteger y promover el desarrollo industrial alemán, especialmente las industrias nacientes, para reducir la brecha que separaba a ese país respecto del Reino Unido de Gran Bretaña" (Neffa, 2000, p. 313). Se preocupación se centraba en las políticas necesarias para que Alemania, en aquella época un país atrasado en comparación con el Reino Unido, acelerase su proceso de industrialización y su crecimiento económico (Freeman 1995).

En este sentido, List (1997) analizó una serie de elementos que ahora forman parte del concepto de sistema nacional de innovación, como las instituciones de educación y entrenamiento, los institutos científicos y tecnológicos, el aprendizaje entre proveedores y consumidores, la acumulación de conocimiento, la adaptación de tecnología importada y la promoción de industrias estratégicas, entre otros, además de proponer políticas alrededor del tema del aprendizaje y el uso de las nuevas tecnologías (Kuramoto, 2007). Al tratar de identificar los rasgos esenciales de una estrategia para alcanzar a los más avanzados, "abogaba por una política tecnológica nacional, muy vinculada a una política industrial y educativa" (Freeman, 1993, p. 124).

\subsubsection{Definiciones de sistema nacional de innovación}

En los inicios de la década de los noventa del siglo XX aparece una serie de literatura que hace énfasis en el rol de los factores particulares de una nación o región para promover el cambio tecnológico, la cual destaca el llamado aprendizaje por interacción, donde usuarios, proveedores, competidores, y la infraestructura científica tecnológica, interactúan en la producción de innovaciones (Cervilla, 2001).

Por consiguiente, la noción de sistema nacional de innovación surge como un modelo interactivo de creación y uso del conocimiento en el cual participan los diferentes agentes 
relacionados con la producción y el desarrollo tecnológico (Rincón, 2004). El sistema nacional de innovación resulta del reconocimiento de la necesidad de estudiar los procesos de innovación bajo una visión sistémica y de la importancia atribuida al contexto institucional y el factor organizacional en el aprendizaje y difusión de tecnologías.

De acuerdo con Pérez (1996), existen dos formas de interpretar la definición de sistema nacional de innovación: una restringida y una amplia. En el sentido estrecho, el sistema nacional de innovación concuerda esencialmente con lo que en América Latina se ha denominado Sistema científico-tecnológico, colocado en muchos casos bajo un Ministerio de Ciencia y Tecnología. Posteriormente, se le ha agregado el término innovación, para señalar, la introducción comercial del progreso técnico, en contraste con las formas académicas de la ciencia y la tecnología. Esto supone que las instituciones públicas se reorientarán a apoyar al sector productivo.

El enfoque estrecho, según Cassiolato (1994) está limitado a las instituciones científicas y técnicas especializadas que tienen que ver con la investigación, el desarrollo y la difusión de las innovaciones, las bibliotecas científicas, los laboratorios de investigación y desarrollo ( I y D), los institutos de normas, las oficinas de patentes, entre otros. Este ha sido el enfoque típico de los estudios de OCDE y de UNESCO, sobre políticas científicas y tecnológicas. La condición más importante de esta aproximación quizás sea la comparación entre distintas instituciones científicas y técnicas en distintos países.

Por otro lado, en el sentido más amplio, el sistema nacional de innovación se entiende como todo aquello que incide en la capacidad innovativa, la actitud innovativa y las posibilidades de innovar en un espacio nacional. Esta visión está relacionada con la concepción amplia de la tecnología, donde el espacio para la innovación tecnológica y para las estrategias competitivas es todo el escenario productivo. En este sentido, esta concepción permite incluir a todos los agentes y todos los espacios relacionados con el progreso técnico en el aparato productivo, al mismo tiempo que la distingue del mundo académico y científico.

Este enfoque más amplio, según Cassiolato (1994) se interesa por todas las instituciones que promueven el aprendizaje y la asimilación de nuevas tecnologías, como también las 
instituciones que se interesan directamente por la investigación y desarrollo. Una idea central de los argumentos de Freeman y Lundvall (citados por Cassiolato, 1994) es que llegar a ser líder en tecnología no es simplemente cuestión de imitar los productos y procesos de los otros. Es también una cuestión de cambios sociales dentro de la industria y dentro de una variedad de otras instituciones. En sus estudios, la organización y el manejo de las relaciones dentro de las empresas, y las redes de colaboración entre empresas, universidades e institutos estatales (especialmente las interacciones usuario/productor/investigador) se consideran importantes para entender por qué este proceso se desarrolla más rápido y eficientemente en algunas áreas que en otras.

Desde la perspectiva de la formulación de políticas tecnológicas, por otro lado, uno de los más importantes ejercicios derivados de la conceptualización de los sistemas nacionales de innovación consiste en las comparaciones internacionales: ¿Qué clase de políticas tecnológicas están concibiendo e instrumentando mis vecinos, socios, rivales o modelos? ¿Cuáles de estas políticas podrían aplicarse domésticamente y a qué costo? (Arocena \& Sutz, 2000).

\subsubsection{Características de los sistemas nacionales de innovación}

En relación al sistema nacional de innovación, siguiendo a Johnson y Lundvall (1994) es relevante señalar dos características fundamentales. En primer lugar, que los sistemas nacionales de innovación conciben a las innovaciones como un proceso social e interactivo en un entorno social específico y sistémico. En segundo lugar, que la atención se centra en los sistemas nacionales (más que en los locales, regionales o transnacionales), por lo tanto, no se deriva de una comprensión meramente teórica, sino de consideraciones sobre historia económica, donde las naciones-Estado son importantes porque permiten la creación de entornos sociales y económicos propicios para los procesos de innovación.

\section{MATERIALES Y MÉTODOS}

ISSN: 1390-9320, Vol. 7, No. 1, febrero 2020 
Este trabajo se realizó bajo la metodología de investigación documental de tipo cualitativa, descriptiva, analítica, reflexiva e interpretativa, cuyo proceso de recolección, localización, pesquisa, análisis y discusión de los documentos bibliográficos seleccionados, incluyó cuatro fases (Hochman y Montero, 1991): 1) focalización de la información, en diferentes fuentes: revistas, artículos, libros, tesis de grado y fuentes académicas; 2) selección de dichas fuentes; 3 ) análisis de los trabajos académicos y 4) interpretación de la información contenida en ellos.

Al respecto, Finol y Nava (1992) consideran que la metodología de la investigación documental facilita el análisis, registro, recuperación y selección de información, puntos de vistas y visiones expuestas por otros autores. Asimismo, se utilizaron diversas herramientas para la búsqueda de revistas académicas-científicas de circulación nacional e internacional en internet (Funes, 2015; Maldonado, 2018): 1) bases de datos internacionales (Web of Science, Scopus, Science Direct) e iberoamericanas (Scielo, Redalyc), 2) motores de búsqueda (Google académico, ResearchGate), 3) directorios temáticos (Latindex, Dialnet) y 4) repositorios y/o redes de bibliotecas virtuales (CLACSO, OEI).

En la búsqueda de información, se usaron dos descriptores, principalmente: transferencia internacional de tecnología y sistema nacional de innovación, considerando tanto literatura a nivel internacional, generalmente en inglés, como iberoamericana, en idioma español. Luego, se realizó un análisis de contenido de los trabajos encontrados. En general, fueron seleccionados los materiales bibliográficos que más se relacionaran con los propósitos de la investigación, tomando en cuenta las posturas de los autores. Para sistematizar, analizar e interpretar cada uno de los trabajos seleccionados en la recopilación, se utilizaron fichas, lecturas detalladas, redacciones preliminares y definitiva. En la última fase, se sintetizó, dedujo y recuperó lo aportes más importantes de los autores para finalmente, discutir sus posiciones, llegar a los resultados y conclusiones.

En general, los pasos seguidos en la elaboración del artículo fueron los siguientes: (a) definición del tema en el grupo de trabajo (b); discusión de su viabilidad y sistematización de los objetivos; (c) búsqueda de materiales bibliográficos teóricos y empíricos a analizar, 
evaluación de la literatura seleccionada a partir de lecturas previas; (d) diseño de fichas de resúmenes y análisis de lecturas; (e) organización de la información, análisis, evaluación y discusión de la misma, y (f) proceso de construcción y redacción del artículo científico (Barrera, 2000; Perona, 2005; Molero, 2010).

\section{ANÁLISIS Y DISCUSIÓN DE RESULTADOS}

En esta parte se analizan y discuten los resultados de la relación entre la transferencia internacional de tecnología y el sistema nacional de innovación en el contexto económico internacional y en países en desarrollo. En este sentido, la literatura sobre desarrollo económico y transferencia de tecnología ha tratado de explicar la relación entre los flujos de tecnología y las características de los sistemas nacionales de innovación, particularmente, las capacidades y políticas para la absorción de tecnologías transferidas (Correa, 1994).

\subsection{Transferencia de tecnología y el sistema nacional de innovación}

Park \& Park (2003, citados por Bartels, Voss, Lederer \& Bachtrog, 2012) definen el sistema nacional de innovación como el perfil estructural y funcional de una nación que determinan su capacidad innovadora y su desempeño económico. Este perfil señala una significativa correlación entre investigación y desarrollo y la estructura industrial, a la par de indicar que la capacidad de recursos es empíricamente significativa para las operaciones de los sistemas nacionales de innovación. Bartels et. al. (2012) han puesto en relieve que las normas, confianza, una acción racional y el grado de autoridad determinan el contenido y estilo del sistema nacional de innovación. De allí que los sistemas nacionales de innovación posean sólidas raíces históricas, las cuales tienden a persistir por largos periodos.

Como la eficiencia y efectividad del cambio tecnológico están resumidas en los sistemas nacionales de innovación, es importante reconocer cuatro nociones básicas siguiendo a Bartels, et al., (2012). En primer lugar, ubicar las innovaciones dentro del desempeño económico competitivo del sistema que están en función de unas relaciones estables y 
cercanas entre los agentes económicos. En segundo lugar, tal relacionamiento está contextualizado por interacciones no de mercado. En tercer lugar, las relaciones e interacciones están diferencialmente acotadas por las especificidades de las 'reglas del juego' institucional y sujetas a fricciones. Y en cuarto lugar, las diferencias en la reserva de conocimiento industrial y en la manera en que ellas son manejadas en términos de política afectan la calidad de los relacionamientos.

Aún más, los sistemas nacionales de innovación, siguiendo a Arocena \& Sutz, (2000), son importantes para analizar y comprender cómo las influencias nacionales intervienen hoy día y podrían afectar futuros productos de la actividad innovadora. Por tales causas, un marco conceptual que facilite el describir las innovaciones, su interpretación y acciones propias es necesario; la noción de sistema nacional de innovación -en cualquiera de sus múltiples definiciones- provee tal marco. Además, el concepto establece un positivo circuito de retroalimentación: fortalecer el sistema nacional de innovación equivale a mejorar el clima innovador y, consecuentemente, la viabilidad de las innovaciones.

El contacto entre estas fuentes y los demandantes de tecnología se desenvuelven a través de un sistema nacional de innovación, el cual impulsa interacciones entre productores y usuarios de tecnología -aquellas instituciones que intervienen en el proceso de aprendizaje de las <<oportunidades técnicas >>- a decir de Arocena \& Stutz (2000). El sistema nacional de transferencia tecnológica puede consistir de varios entes. El más importante de ellos lo constituyen los innovadores (creadores de tecnología), luego se encuentra a los comercializadores (compañías) y a las instituciones del gobierno central. Gurbiel (2002) ofrece un esquema de participantes en el proceso de transferencia tecnológica asociada con los sistemas nacionales de innovación que se reproduce a continuación en Gráfica 1. 
Gráfica 1. Transferencia de tecnología y participantes del sistema nacional de innovación

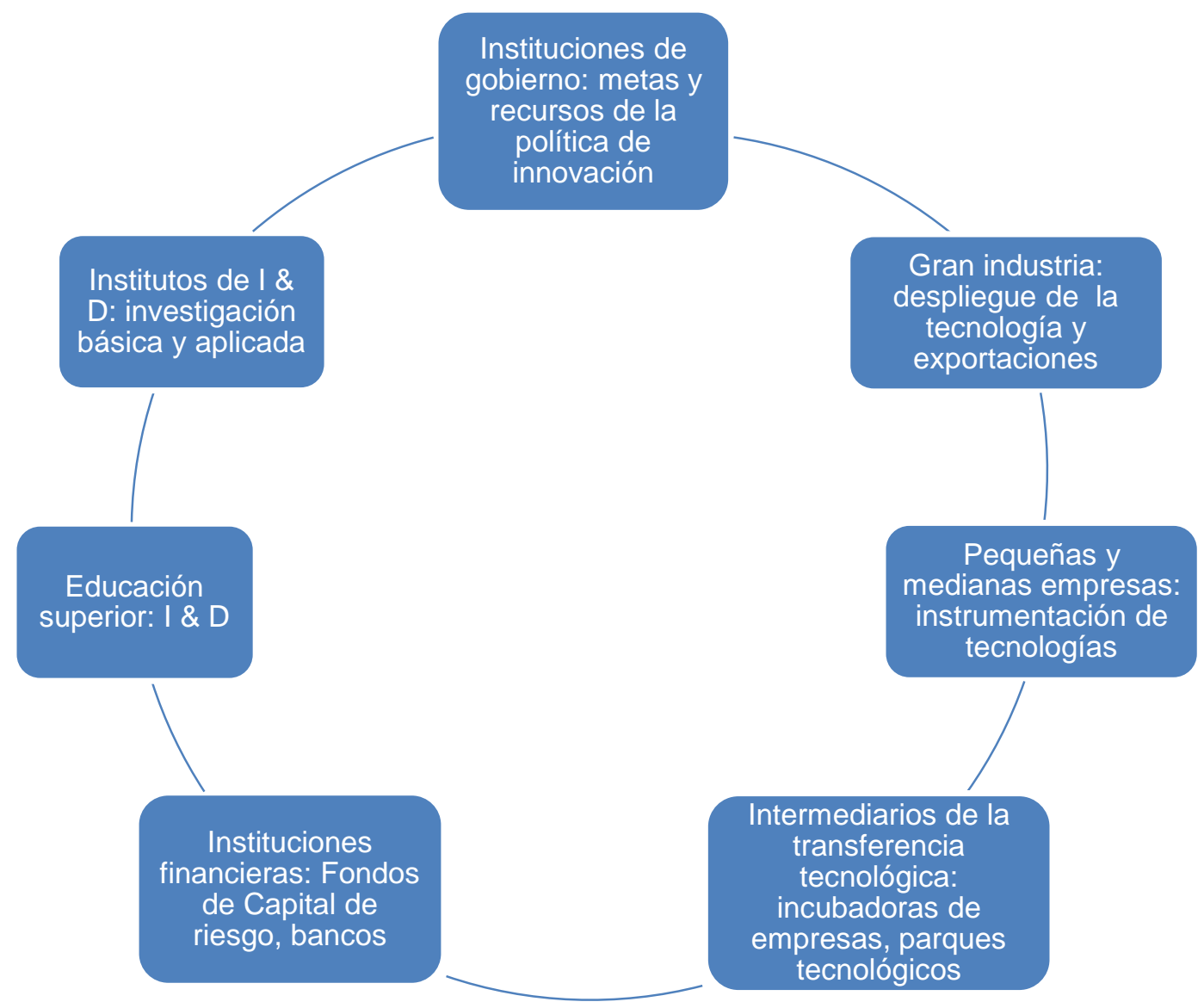

Fuente: Gurbiel (2002).

A la izquierda del sistema, se ubican los generadores de tecnología, entre ellos: las universidades, institutos de tecnología y otras instituciones de educación superior, públicas y privadas, quienes se desenvuelven en el área de la investigación básica o aplicada, por iniciativa propia, o mediante mecanismos de financiamiento privado, donde los bancos y otras instituciones financieras cumplen un papel fundamental dentro de una economía de circuito.

A la derecha del sistema, se ubica el complejo industrial y comercial (y eventualmente, militar) que adquiere las innovaciones producidas, tanto a lo externo de ellas, como dentro de las firmas. Gurbiel (2002) señala que la transferencia interna de tecnología es aquella conducida preferiblemente dentro de una entidad individual o entre sus afiliadas. Tal ente 
actúa en ese caso como creador/innovador y usuario. El alcance del proceso interno es limitado por la disponibilidad de recursos internos de investigación y desarrollo, así como las capacidades de su instrumentación.

En lo que respecta al sector público e instituciones del gobierno central, además de su papel tradicional de formulador de políticas y de emisor de recursos presupuestarios para la innovación, Constantini \& Liberati (2014) enfatiza -al relacionar el sector público con el marco institucional nacional para la transferencia tecnológica- que su influencia pasa por los significativos impactos del consumo del gobierno, especialmente cuando los recursos están concentrados en la salud, educación; lo que sugiere que el gasto público puede estimular los logros del desarrollo humano, y por el positivo impacto del patrimonio infraestructural.

De esta manera, los procesos de innovación tecnológica no se reducen a la capacidad que posean las empresas, laboratorios, el sector público, sino que necesitan de un sistema nacional de innovación, por cuanto se requiere un conjunto de agentes involucrados y sus relaciones mutuas. De allí que un mayor dinamismo tecnológico solo puede originarse en la sociedad si en ésta coexisten las infraestructuras y redes necesarias para apoyar la actividad innovadora, los procesos de generación, transferencias, adaptación y difusión de tecnologías (Martínez, 1994).

Como punto de partida para el proceso de transferencia tecnológica, la visión neoschumpeteriana enfatiza que, a nivel macroeconómico, lo que importa es el momento en que las innovaciones radicales comienzan a ser aplicadas en masa dentro de los sectores de rápido crecimiento dentro de la economía, y dentro de tal época, el sistema socioinstitucional juega un papel preponderante en paralelo al sistema tecnoeconómico propiamente dicho. Golichenko (2016) expresa que mientras el sistema socioinstitucional no esté preparado para aceptar el nuevo estilo tecnológico por hallarse atado al antiguo orden se forma una barrera para la diseminación de las innovaciones. Solo cuando cierta coherencia y armonía son alcanzadas entre el sistema tecnoeconómico y el socioinstitucional las innovaciones se difunden rápidamente, son incrementalmente 
mejoradas, y el proceso de destrucción creativa del anterior orden tecnológico incrementa su velocidad.

En el origen del proceso de transferencia y comercialización de innovaciones tecnológicas se hallan las invenciones o descubrimientos. Las invenciones pueden originarse dentro o fuera de los límites de la firma. Datta, Mukherjee \& Jessup (2014) revelan seis principales fuentes de invención: 1) creatividad organizacional, 2) investigación y desarrollo, 3) alianzas y colaboraciones, 4) motores de innovación, 5) clusters tecnológicos y 6) los "derrames" de tecnología (technology spillovers, que pueden ocurrir cuando las firmas transfieren tecnología a proveedores locales en aras de incrementar la calidad de los insumos a menores precios). Para las formas de derrames tecnológicos consultar Liang (2016) y Newman, Rand, Talbot \& Tarp (2015).

\subsection{Los sistemas nacionales de innovación en el contexto global}

El escenario económico internacional desde fines de los años ochenta y durante los noventa del siglo XX y lo que va del siglo XXI ha estado caracterizado por un creciente proceso de globalización, constatable en el explosivo crecimiento de los flujos financieros internacionales, la inversión extranjera directa, el comercio internacional e intrarregional y el intercambio de tecnología. En forma paralela, se fue conformando un nuevo paradigma tecno-económico basado principalmente en las innovaciones de la microelectrónica, así como de la biotecnología, los nuevos materiales y las energías renovables, potenciando, además, factores organizacionales dentro de las propias empresas y entre éstas y sus pares (Pitalluga, 2000).

Esas dos tendencias confluyeron, determinando el predominio de un patrón mundial de competencia en el cual los factores de éxito económico de las firmas de los distintos sectores productivos se conforman en el ámbito mundial. Por otra parte, el tema del cambio tecnológico y sus implicaciones para la competividad y el comercio internacional ha adquirido una importancia creciente dentro de la literatura de la ciencia económica (Correa, 1994). Esta visión está basada en diversas perspectivas teóricas. En términos generales, 
las nuevas bases de la competitividad de las empresas dependen cada vez más de las ventajas construidas a través de la innovación tecnológica, superando las ventajas comparativas derivadas de la dotación de los factores productivos o de los recursos naturales (Pitalluga, 2000).

A un meta-nivel de análisis, Archibugi y lammarino (1999, citados por Bartels et al., 2012) desarrollan una taxonomía de la globalización de la innovación con categorías, acciones y formas como dimensiones, señalando que la colaboración global tecno-científica se lleva a cabo por medio de los componentes de los sistemas nacionales de innovación, de universidades, centros de investigación pública, empresas nacionales e internacionales. Estos también constituyen los actores en el modelo neoevolucionista de triple hélice universidad-industria-gobierno de Leydesdorff (2001, citado por Bartels et al., 2012). Este modelo con dimensiones funcionales e institucionales refleja la arquitectura central de los sistemas nacionales de innovación.

Por otro lado, varios estudios citados por Malikane \& Chitambara (2017) recalcan que una apertura comercial brinda al país anfitrión mayor acceso a las tecnologías foráneas. En este sentido, concluyen que esta apertura tiene un positivo y significante impacto en la productividad del trabajo. Estos resultados sugieren que las políticas de mercado abierto son cruciales para los países en desarrollo en orden de atraer tecnología. Pero solo la apertura no es suficiente para alcanzar una mayor capacidad de absorción. En los países en desarrollo, la adquisición de tecnología a menudo equivale a la adaptación de métodos existentes a circunstancias locales. Una gradual adopción de nuevas técnicas o inputs puede ser óptima para productores reacios al riesgo respecto a costosas inversiones y retornos inciertos. Los productores precisan aprender cómo aplicar la nueva tecnología y mejorarla gradualmente a través del tiempo. Las naciones tienden a adquirir tecnología internacional más fácilmente si las firmas domésticas poseen programas de investigación y desarrollo, si existen universidades y laboratorios de investigación a lo interno, o la presencia de una base sólida de habilidades técnicas y de capital humano (Hoekman et al., 2005). 
Franco, Montresor \& Vittucci (2011), reconocen que el comercio internacional es un medio extremadamente importante de transferencia tecnológica. Al importar, la economía puede obtener un doble beneficio tecnológico. Primero que todo, se beneficia de las inversiones en investigación y desarrollo del foráneo, cuyos resultados están incorporados en las mercancías que vende en el extranjero. Segundo, el país de acogida también se beneficia de las inversiones en investigación y desarrollo realizadas por otros países del extranjero, con los cuales aquél inicialmente ha comercializado, esto en términos de derrames indirectos de investigación y desarrollo.

De cara a políticas específicas, la evidencia sugiere que los países que aplican medidas de comercio a puertas cerradas fallan en alcanzar los beneficios implícitos de la tecnología dentro del comercio internacional, por lo que Hoekman et al. (2005) argumentan por políticas librecambistas como una prioridad, tan importante como unas sólidas políticas generales de tecnología. Estos autores señalan que importaciones de bienes intensivos en capital provenientes de países de ingresos altos están asociadas con una mayor productividad total de los factores de los países en desarrollo. Adicionalmente, los derrames procedentes de importaciones intensivas en tecnología pueden incrementar las exportaciones porque los exportadores precisan emplear tecnologías que impulsen niveles y patrones de calidad requeridos internacionalmente.

En términos generales, Hoekman et al. (2005) arguyen como prioritario en todos los tipos de países en desarrollo el establecer políticas efectivas de tecnología, que incluyen el mejoramiento de la educación básica, construcción de infraestructura apropiada y la reducción de barreras de entrada para las firmas locales que podrían ser suplidoras de las multinacionales, siendo ésta una compleja tarea que involucra el desarrollo de capital humano, la protección de los derechos de propiedad intelectual y expandir el sistema nacional de innovación.

\subsection{Los sistemas nacionales de innovación y las políticas de innovación en países en desarrollo: características y fortalecimiento}


En contraste con la situación que presentan los países industrializados, los países de América Latina y de manera similar otros países en desarrollo, aun no han podido ampliar sus sistemas de investigación y desarrollo comparables a los de los países desarrollados, ya sea por las características mismas de los sectores productivos que orientan la producción nacional, o por el enfoque de las políticas de fortalecimiento de los sistemas de innovación (Morales, Ortiz y Arias, 2012).

Por otra parte, en los países en desarrollo, sobre todo en América Latina se dio el fortalecimiento de la ciencia, tecnología e innovación, pero con una década de retraso o algo más, en comparación con los países más desarrollados (Ekboir y Parellada, 1999; Salazar, 2010). Sin embargo, a pesar de que aun no han logrado procesos de innovación en la misma proporción que lo han realizado los países industrializados, la necesidad creciente de adaptar las condiciones actuales a los requerimientos del mercado y de la misma sociedad, ha hecho que la innovación pueda llegar a tener fuentes diferentes, pues como señalan Ekboir y Parellada (1999), Anlló y Suárez (2008) y Hernández (2010), los sectores de baja y media tecnología que en su mayoría componen la estructura productiva de los países emergentes, desarrollan innovaciones a partir de la incorporación de nuevas tecnologías importadas que adaptan a sus necesidades. No obstante, a pesar de la poca existencia de departamentos de investigación y desarrollo o la creación de tecnologías de punta, no obstante, pueden darse innovaciones a nivel adaptativo e incremental (Morales, et. al., 2012).

La sofisticación del sistema nacional define los límites y las posibilidades de las políticas de innovación, determinando la efectividad de las mismas. Dentro de estas políticas, una de las decisiones más importantes es el balance entre los desarrollos tecnológicos propios y la imitación o incorporación de conocimientos generados en otros países. Los países con sistemas nacionales de innovación débiles dependen casi exclusivamente de las tecnologías desarrolladas en otros países. En estos países, las inversiones en actividades formales de investigación y desarrollo tienen poca influencia sobre las tasas de crecimiento económico, mientras que las inversiones en educación y entrenamiento parecen jugar un papel mucho más importante (Ekboir y Parellada, 1999). 
Una de las explicaciones de este fenómeno es que existen programas de educación y entrenamiento que les permiten absorber el acervo internacional de conocimientos científicos y tecnológicos. Los sistemas de investigación débiles solo pueden realizar actividades simples de investigación estratégica (es decir, desarrollos menores). Por lo que para realizar tareas más complejas es necesario fortalecer el sistema de innovación por medio de inversiones importantes y sostenidas por periodos largos de tiempo, hasta alcanzar la masa crítica a partir de la cual se logren instituciones de investigación y desarrollo productivas. Por consiguiente, las inversiones destinadas a crear conocimientos originales solo son beneficiosas en el largo plazo, y siempre y cuando se den las condiciones para que el conocimiento formal pueda transformarse en una innovación.

Estas condiciones incluyen un mercado de capitales eficiente, creación de instrumentos financieros que apoyen a la innovación, infraestructura, instituciones y legislación adecuados, condiciones que a menudo faltan en los países en desarrollo. A partir de un cierto nivel de desarrollo, la estrategia de importar tecnología sin un esfuerzo interno de adaptación se vuelve inefectiva (Exboir y Paralleda, 1999).

Al llegar a ese estado, una condición necesaria para el desarrollo es el fortalecimiento del sistema nacional de innovación, dado que es la única forma en la cual se pueda absorber tecnologías más sofisticadas que aumenten la competitividad. La capacidad del sistema nacional de innovación puede construirse por medio de políticas de largo plazo de fortalecimiento institucional, de incentivos a la investigación en empresas privadas, de inversión en recursos humanos y en infraestructura de investigación y en la creación de instrumentos que promuevan las colaboraciones interinstitucionales en investigación y desarrollo (Ekboir y Paralleda, 1999).

Por tanto, uno de los elementos más importantes de estas políticas es la continuidad, pues instituciones que se han construido a lo largo de muchos años pueden destruirse rápidamente si no se mantienen los incentivos adecuados. A medida que el sistema nacional de innovación se fortalece, se amplía el espectro de investigaciones estratégicas que puede realizar. Eventualmente, puede alcanzar un nivel de sofisticación elevado y 
comenzar a realizar algunas investigaciones motivadas en la curiosidad. Una vez alcanzado este nivel, el país ya no depende únicamente de las tecnologías importadas, sino que puede desarrollar tecnologías propias. Cuanto más fuerte es el sistema nacional de innovación de un país, más importante se vuelve la prospección tecnológica para identificar líneas de investigación promisorias.

\section{CONCLUSIONES}

Una adecuada transferencia de tecnologías ha de tener su base en un sistema nacional de innovación debidamente implantado y con producción propia, ello constituye el inicio del proceso de derrame y expansión de saberes que, desde la localidad o el establecimiento aislado, se propaga hacia las firmas externas, generando un aumento de las reservas internacionales, además de provocar el reconocimiento de la comunidad mundial.

Este sería el recorrido más adecuado para generar crecimiento económico y desarrollo de las fuerzas productivas, pero también los procesos de transferencia tecnológica hacia adelante, los que se inician con una inversión de firma extranjera, podrían generar un "refrescamiento" de los procesos tecnológicos internos que, al encontrarse con un sistema nacional consolidado, potenciarían los encadenamientos y los efectos multiplicadores de la inversión interna, especialmente aquella destinada a robustecer el capital humano local, lugar donde los institutos universitarios han de desempeñar un papel de primera línea.

Es necesario recalcar que la producción de tecnologías en productos y, aun más, en procesos, resulta esencial para superar el estado estacionario en que parece ubicarse la economía latinoamericana, caracterizada por la dificultad para generar empleos de calidad, con salarios dignos y con prestaciones de retiro suficientes para asegurar un tipo de sustentabilidad intergeneracional que mantenga el patrón de consumo altamente tecnificado de estas sociedades.

Solo mediante la generación autóctona de tecnologías aplicables a la inmensa diversidad biológica y cultural de la región latinoamericana se podrá superar los problemas seculares 
de concentración del ingreso, fuga de capitales (humanos y financieros) hacia el Primer Mundo, la depauperación de las clases medias, el atraso educativo para el avance científico, la debilidad de los mercados internos y la omnipresente dependencia: cultural, económica, financiera y tecnológica.

Uno de los obstáculos al avance científico y tecnológico en países de la periferia pueden derivarse de ciertas exigencias de la comunidad empresarial y los centros financieros globales, específicamente los derechos de propiedad intelectual que, luego de procesar bienes inmateriales-culturales o recursos naturales inexplotados en la nación en desarrollo, son transformados en mercancías de alto valor agregado en el mundo altamente industrializado, generando un hiato insalvable al no compartir el avance científico-técnico que ha hecho posible el aprovechamiento del recurso primario, profundizando la pérdida de divisas y la fuga de talentos.

Los más elevados intereses de los préstamos para la investigación y desarrollo tecnológico a nuestras economías, en comparación con los grandes conglomerados industriales presentes en el Primer Mundo, mantienen una postura conservadora hacia la investigación científico-técnica por parte de gobiernos y empresarios locales, siempre ávidos de rápidos retornos del capital invertido y que, frente a una situación política marcada por marchas y contramarchas en los procesos de integración económica y monetaria, no materializan formas autónomas de generación, comercialización y compartición del "saber hacer" localsectorial que constituyen la médula del progreso y expansión económica, paso necesario para el progreso económico sostenible, ya que el mantenimiento del atraso productivo, el rezago tecnológico y la pobreza son los destructores efectivos del ambiente y las principales amenazas para las generaciones por venir.

Por consiguiente, el sistema nacional de innovación ha de ser fortalecido, subvencionado en forma conjunta por los sectores público y privado que financien el despegue tecnológico (catch-up), con participación comunitaria y de los poseedores del saber local y ancestral, una verdadera comunidad científica y tecnológica, formada desde la escuela inicial e impulsada por los acuerdos que a nivel de los organismos internacionales, tales como, por 
ejemplo, la Organización de Naciones Unidas (ONU), han de reimpulsarse para incluir el cierre de la brecha tecnológica y digital como uno de los objetivos del nuevo milenio.

\section{REFERENCIAS BIBLIOGRÁFICAS}

Anlló, G. y Suárez, D. (2008). Innovación: algo más que I+D. Evidencias iberoamericanas a partir de las encuestas de innovación: construyendo las estrategias empresarias competitivas. Recuperado el 17 de octubre de 2019. http://www.oei.es/salactsi/innova.pdf.

Arocena, R. \& Sutz, J. (2000). Looking at national systems of innovation from the south. Industry and Innovation, Volumen 7 (1), 55-75. Recuperado el 18 de mayo de 2019.

https://www.researchgate.net/publication/227616191 Looking at National System of Innovation from the South.

Ávalos, I. (1994). Transferencia de tecnología. En: Martínez, E. (Ed.). Ciencia, tecnología y desarrollo. Interrelaciones teóricas y metodológicas. Caracas: Editorial Nueva Sociedad. 411-454.

Barrera, M. (2000). Sugerencias para redactores, comunicadores e investigadores. Caracas: Fundación Sypal.

Bartels, F., Voss, H., Lederer, S. \& Bachtrog, Ch. (2012). Determinants of national innovation systems: policy implications for developing countries. Innovation: Management, Policy \& Practice, Volumen 14 (1), 2-18. Recuperado el 20 de julio de 2019. https://www.researchgate.net/publication/271152270 Determinants of National In novation Systems Policy implications for developing countries. 
Cassiolato, J. (1994). Innovación y cambio tecnológico. En: Martínez, E. (Ed). Ciencia, tecnología y desarrollo. Interrelaciones teóricas y metodológicas. Santiago: Editorial Nueva Sociedad. 261-305.

Cervilla, M. (2001). La innovación como un proceso económico y social: algunas implicaciones para el diseño de una estrategia de desarrollo. Caracas: Centro de Estudios del Desarrollo. Universidad Central de Venezuela.

Costantini, V. \& Liberati, P. (2014). Technology transfer, institutions and development. Technological Forecasting \& Social Change. Volumen 88 (1), 26-48. Recuperado $\begin{array}{lllll}\text { el } & 16 & \text { de } & \text { septiembre }\end{array}$ https://www.researchgate.net/publication/254449632 Technology transfer institutio ns and development.

Correa, C. (1994). El nuevo escenario para la transferencia de tecnología: repercusiones en los países en desarrollo. Comercio Exterior. Volumen 44 (9), septiembre, 746758.

Datta, A., Mukherjee, D. \& Jessup, L. (2014). Understanding commercialization of technological innovation: taking stock and moving forward. R\&D Management, Volumen 45 (3), 215-249. Recuperado el 19 de julio de 2019. https://www.researchgate.net/publication/261719373 Understanding commercializ ation of technological innovation Taking stock and moving forward.

Escorsa, P. y Valls, J. (2001). Tecnología e innovación en la empresa. Dirección y gestión. Bogotá: Editorial Alfaomega.

Finol, T. y Nava, H. (1992). Procesos y productos en la investigación documental. Maracaibo: Universidad del Zulia. 
Exboir, J. y Parellada, G. (1999). Algunas reflexiones respecto a los sistemas de innovación en la era de la globalización. Documento de trabajo No. 9. Recuperado el 25 de octubre de 2019. https://inta.gob.ar/sites/default/files/scripttmp-dt 09.pdf.

Franco, C., Montresor, S. \& Vittucci, M. (2011). On indirect trade-related R\&D spillovers: the average propagation length of foreing R\&D. Structural Change and Economic Dynamics. Volumen 22 (3), 227-237. Recuperado el 22 de octubre de 2019. https://www.sciencedirect.com/science/article/abs/pii/S0954349X11000385.pdf.

Freeman, Ch. (1993). La experiencia de Japón. El reto de la innovación. Caracas: Editorial Galac.

Freeman, Ch. (1995). The 'national system of innovation' in historical perspective. Cambridge Journal of Economics. (19), 5-24. Recuperado el 25 de septiembre de 2019.

http://www.forschungsnetzwerk.at/downloadpub/1995 Freeman NSI historial per spective.pdf.

Funes, C. (2015). Bases de datos para la investigación en economía. Serie Bibliotecología y Gestión de Información. (94). Santiago: Departamento de Gestión de Información. Universidad Tecnológica Metropolitana. Recuperado el 30 de octubre de 2019. http://eprints.rclis.org/25275/1/serie\%2094\%202.ed.economia.pdf.

Golichenko, O. (2016). The national innovation system. From concept to research methodology. Problems of Economic Transition. Volumen 58 (5), 463-481. $\begin{array}{llllll}\text { Recuperado el } 27 \text { de octubre } & 2019 .\end{array}$ https://www.tandfonline.com/doi/abs/10.1080/10611991.2016.1225452. 
González, V., Clemenza, C. y Ferrer, J. (2008). Estrategias de transferencia tecnológica entre las universidades públicas. Maracaibo: Consejo de Fomento, Vice Rectorado Administrativo, Universidad del Zulia.

Gurbiel, R. (2002). Impact of innovation and technology transfer on economic growth: the Central and Eastern Europe experience. Warsaw School of Economics Center of International Production Cooperation. Recuperado el 16 de Julio de 2019. https://www.eadi.org/typo3/fileadmin/WG Documents/Reg WG/gurbiel.pdf.

Hernández, I. (2010). Una conceptualización de la innovación en economías emergentes. En: Bejarano, P., Zerda, A. y Cortés, C. (Eds). Innovación. Desafíos para el desarrollo en el siglo XXI. Bogotá: Universidad Nacional de Colombia. 51-63.

Hochman, E. y Montero, M. (1991). Técnicas de investigación documental. México: Editorial Trillas.

Hoekman, Bernard; Maskus, Keith \& Saggi, Kamal (2004). Transfer of technology to developing countries: unilateral and multilateral policy options. World Development. Volumen 33 (10), 1587-1602. Recuperado el 24 de agosto de 2019. https://www.sciencedirect.com/science/article/abs/pii/S0305750X05001245.pdf.

Johnson, B. y Lundvall, B. (1994). Sistemas nacionales de innovación y aprendizaje institucional. Comercio Exterior. 44 (8), 695-704. Recuperado el 17 de octubre de 2019. http://revistas.bancomext.gob.mx/rce/magazines/362/4/RCE4.pdf.

Kuramoto, J. (2007). Sistema de innovación tecnológica. En: Grupo de Análisis para el Desarrollo-GRADE. Investigación, políticas y desarrollo en el Perú. Lima. 103133. Recuperado el 25 de noviembre de 2019. http://biblioteca.clacso.edu.ar/Peru/grade/20100513020845/InvPolitDesarr-3.pdf. 
Liang, F. (2017). Does foreign direct investment improve the productivity of domestic firms? Technology spillovers, industry linkages, and firm capabilities. Research Policy. Volumen 46, (1), 138-159. Recuperado el 5 de agosto de 2019. https://ideas.repec.org/a/eee/respol/v46y2017i1p138-159.html.

List, F. (1997). Sistema nacional de economía política. México: Fondo de Cultura Económica.

Lutteral, P. (2016). La transferencia internacional de tecnología. Desafíos, tratamiento tributario internacional y propuestas para la redacción de contratos. Maestría en Comercio Internacional. Universidad de Barcelona. Recuperado el 4 de agosto de 2019. https://rdu.unc.edu.ar/bitstream/handle/11086/6056/Lutteral\%2C\%20Patricio.\%20L a\%20Transferencia\%20Internacional\%20de\%20Tecnolog\%C3\%ADa....pdf? seque nce $=1$ \&isAllowed $=y$.

Maldonado, Á. (2018). Directorios, clasificaciones y rankings de revistas científicas. Madrid: Centro de Ciencias Humanas y Sociales, Consejo Superior de Investigaciones Científicas. Recuperado el 30 de octubre de 2019. https://digital.csic.es/bitstream/10261/172769/1/DirectoriosRankingsRevistasCientif icasFichasResumenoct2018.pdf.

Malikane, C \& Chitambara, P. (2017). Foreign direct investment (FDI), productivity and the technology gap in African economies. Journal of African Trade. Volumen 4, (1-2), 61-74. Recuperado el 5 de agosto de 2019. https://www.sciencedirect.com/sdfe/reader/pii/S2214851517300191/pdf.

Martínez, E. (1994). Glosario En: Martínez, E. (Ed.). Ciencia, tecnología y desarrollo. Interrelaciones teóricas y metodológicas. Caracas: Editorial Nueva Sociedad. 511-522. 
Molero, L. (2010). Guía para la redacción de artículos científicos en las ciencias humanas y sociales. Maracaibo: Fundacite Zulia.

Morales, M.; Ortíz, C. y Arias, M. (2012). Factores determinantes de los procesos de innovación: una mirada a la situación en Latinoamérica. Revista de la Escuela de Administración y Negocio. (72), 148-163. Recuperado el 15 de octubre de 2019. http://www.scielo.org.co/pdf/ean/n72/n72a10.pdf.

Neffa, J. (2000). Las innovaciones científicas y tecnológicas. Una introducción a su economía política. Buenos Aires: Editorial Lumen / Hvmanistas.

Newman, C., Rand, J., Talbot, T. \& Tarp, F. (2015). Technology transfers, foreign investment and productivity spillovers. European Economic Review. Volumen 76 (2), 168-187. $\begin{array}{llllll}\text { Recuperado el de } & 5 & \text { de } & \end{array}$ https://www.sciencedirect.com/sdfe/reader/pii/S0014292115000367/pdf.

Pérez, C. (1996). Nueva concepción de la tecnología y el sistema nacional de la innovación. Cuaderno del CENDES. Volumen 13 (31), 9-33. Recuperado el 15 de agosto de 2019.

http://dev1.carlotaperez.org/downloads/pubs/CENDES Nva concep de Tech y SNI 1996.pdf.

Perona, E. (2005). Lineamientos generales para escribir un paper o trabajo de investigación. Recuperado el 30 de octubre de 2019. http://portal.eco.unc.edu.ar/files/deconomia/Lineamientos\%20generales\%20para\% 20escribir\%20un\%20paper\%20DOC\%2027.pdf.

Pineda, Julio; Duarte, Astrid; Ponce, César; Guzmán, Rubén y Huaca, José (2016). Modelo de transferencia de tecnología ecuatoriano: una revisión. Congreso Internacional de Información. Recuperado el 7 de agosto de 2019. https://www.researchgate.net/publication/311562780 Modelo de transferencia de 
tecnologia ecuatoriano una revision Ecuadorian model of technology transfer a review.

Pitalluga, L. (2000). Cambios tecnológicos recientes: nuevos enfoques y hechos estilizados. Documento de trabajo. Recuperado el 15 de octubre de 2019. https://www.colibri.udelar.edu.uy/jspui/bitstream/20.500.12008/4234/5/dt-10-00.pdf.

Rincón, É. (2004). El sistema nacional de innovación: un análisis teórico-conceptual. Opción. Diciembre, (45), 94-117.

Rincón, É. (2008). Enfoques teóricos y perspectivas de análisis de la innovación y el cambio tecnológico. Ensayos sobre la economía del cambio tecnológico. Trabajo de Ascenso para optar a la categoría de Profesor Titular. Maracaibo: Universidad del Zulia.

Rincón, É. (2010). Fundamentos económicos del cambio tecnológico desde la perspectiva del desarrollo. Trabajo de Grado para optar al Título de Magíster Scientiarum en Planificación y Gerencia de Ciencia y Tecnología. Maracaibo: Universidad del Zulia.

Rivera, M. y Caballero, R. (2003). Los sistemas de innovación nacionales y la teoría del desarrollo. Problemas del desarrollo. Volumen 34 (134), 9-31. Recuperado el 17 de octubre de 2019. https://www.redalyc.org/pdf/118/11825944002.pdf.

Salazar, M. (2010). Una aproximación a la innovación desde la evolución de la política de ciencia y tecnología. En: En: Bejarano, P., Zerda, A. y Cortés, C. (Eds). Innovación. Desafíos para el desarrollo en el siglo XXI. Bogotá: Universidad Nacional de Colombia. 91-110.

Sánchez, Y. (2016). La transferencia de tecnología en el sudeste asiático y su impacto en el desarrollo socioeconómico. La Habana: Centro de Investigaciones de 
Economía Internacional. Recuperado 11 de julio de 2019. http://biblioteca.clacso.edu.ar/Cuba/cieiuh/20161003123934/LatransferenciadetecnologiaenelSudesteasiaticoysuimpactoe neldesarrollosocioeconomico.pdf. 